\title{
Why much of the Atlantic circulation enters the Caribbean Sea and very little of the Pacific circulation enters the Sea of Japan
}

\author{
Doron Nof * \\ Department of Oceanography 4320 and the Geophysical Fluid Dynamics Institute, The Florida State \\ University, Tallahassee, FL 32306-4320, USA
}

\begin{abstract}
A peculiar aspect of western marginal seas is that some of them accommodate large transports whereas others, apparently similar seas, accommodate relatively small transports. For instance, about 30-40 Sv of Atlantic upper water passes through the Caribbean and yet only $2 \mathrm{~Sv}$ or so passes through the Sea of Japan. The passages connecting the Caribbean to the Atlantic are somewhat larger than their Sea of Japan counterparts, but the dimensions of the gaps are too similar to each other to account for the large difference in the transports.

Four different baroclinic models are used to investigate the problem. First, we used a winddriven, layer-and-a-half isopycnal model with an idealized basin and an adjacent marginal sea separated from the main basin by a thin and long island. In this scenario, thermocline water enters the marginal sea via a gap to the south of the island and exits the sea via a gap to the north of the island. The mean latitudinal position of the marginal sea is gradually increased and the numerical transport across the sea is measured. We then compared the transport determined this way to that computed using three analytical baroclinic models: Godfrey's island rule (Godfrey, J. S. (1989). A Sverdrup model of the depth-integrated flow for the world ocean allowing for island circulations. Geophysical and Astrophysical Fluid Dynamics, 45, 89-112.), the ' $\beta$-controlled' transport formula (Nof, D. (1993). The penetration of Kuroshio water into the Sea of Japan. Journal of Physical Oceanography, 23, 797-807), and the general gap transport formula (Nof, D., (1995). Choked flows and wind-driven interbasin exchange. Journal of Marine Research, 53, 23-48). The results were also compared to Minato and Kimura's (Minato, S., \& Kimura, R. (1980). Volume transport of the western boundary current penetrating into a marginal sea. Journal of the Oceanographic Society of Japan, 36, 185-195) barotropic calculations.
\end{abstract}

All four baroclinic models show that, when the marginal sea is situated in low and mid

* Fax: +1-904-644-2581.

E-mail address: nof@ocean.fsu.edu (D. Nof). 
latitude, the penetration of thermocline water into the marginal sea decreases rapidly with increased latitude. By contrast, in equatorial marginal seas, the penetration of thermocline water increases with increasing latitude. Minato and Kimura's (1980) barotropic model shows a similar behavior except that the maximum transport occurs at a much higher latitude. Excellent agreement between the numerics and Godfrey's island rule formula is found in equatorial seas. As the latitude of the marginal sea increases to low and mid latitudes, the deviations from the numerics increase dramatically though the agreement is still very reasonable.

Both the $\beta$-controlled formula and the gap formula are relatively accurate in mid latitudes when the sea is close to the separation latitude. The importance of frictional forces within the western boundary current results in the $\beta$-controlled formula failing for marginal seas situated far away from the separation latitude. Similarly, the general gap formula fails in equatorial latitudes where there is no western boundary current south of the island.

On the basis of all four baroclinic models, it is suggested that the reason for the small transport within the Sea of Japan (relative to the Caribbean) is its relatively high latitude and not the size of the gaps connecting it to the Pacific Ocean (or frictional effects). (C) 2000 Elsevier Science Ltd. All rights reserved.

\section{Contents}

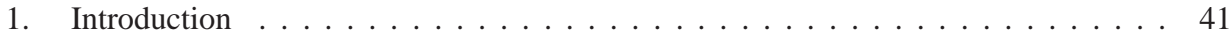

1.1. Classification ......................... 43

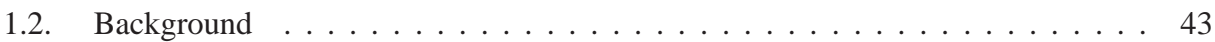

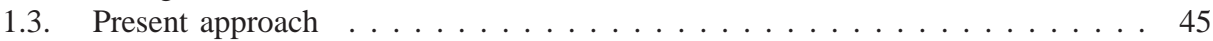

2. Numerical simulations . . . . . . . . . . . . . . . . . . . . . . . . 48

2.1. The numerical model . . . . . . . . . . . . . . . . . . . 49

2.2. Results ........................... 50

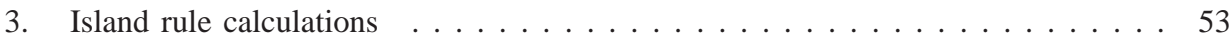

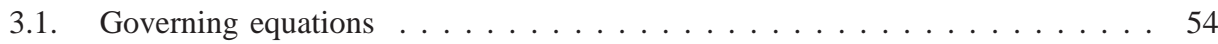

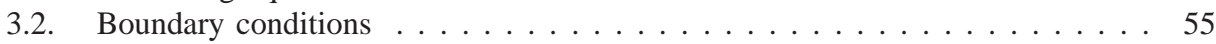

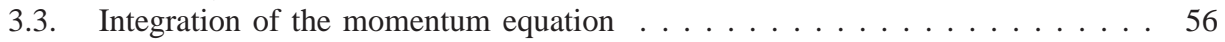

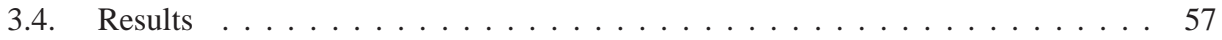

4. The ' $\beta$-controlled' gap formula $\ldots \ldots \ldots \ldots \ldots \ldots \ldots$

5. General gap formula . . . . . . . . . . . . . . . . . . . . 59

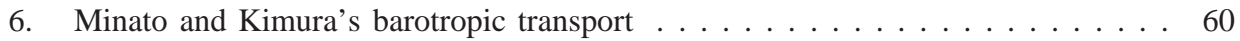

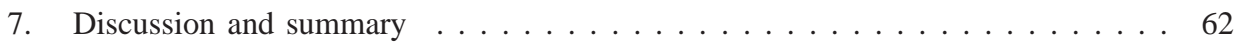

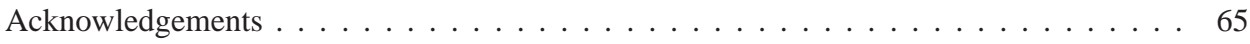

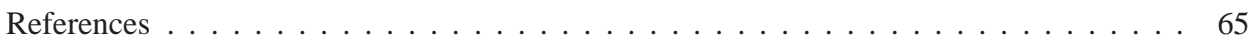




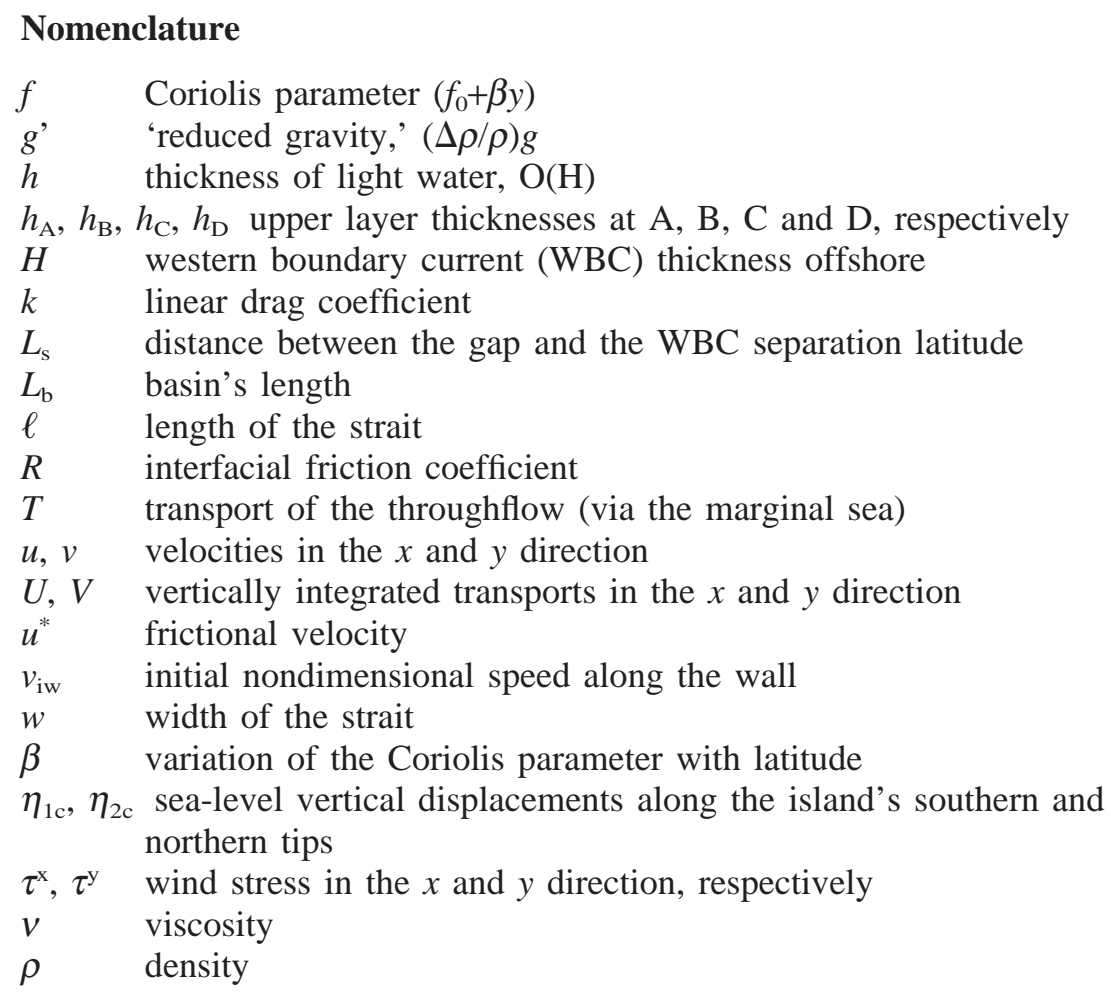

\section{Introduction}

Understanding the exchange of water between oceans and marginal seas is important to our understanding of the general circulation within the ocean. For reasons that are not presently clear, some marginal seas exchange large amounts of water with their adjacent oceanic basins (e.g., the Caribbean-Gulf of Mexico system) whereas others (e.g., the Sea of Japan) exchange small amounts of water with their neighboring bodies of water (Fig. 1). The purpose of this study is to examine these peculiar variations in the basin-marginal sea exchange process. We shall show that, as long as the gaps are of the order of the Rossby radius, their size has very little to do with the amount of water being exchanged. Rather, it is the latitude of the gaps that plays the most crucial role in the exchange process. We begin our presentation by introducing the various kinds of gaps and exchange processes. 

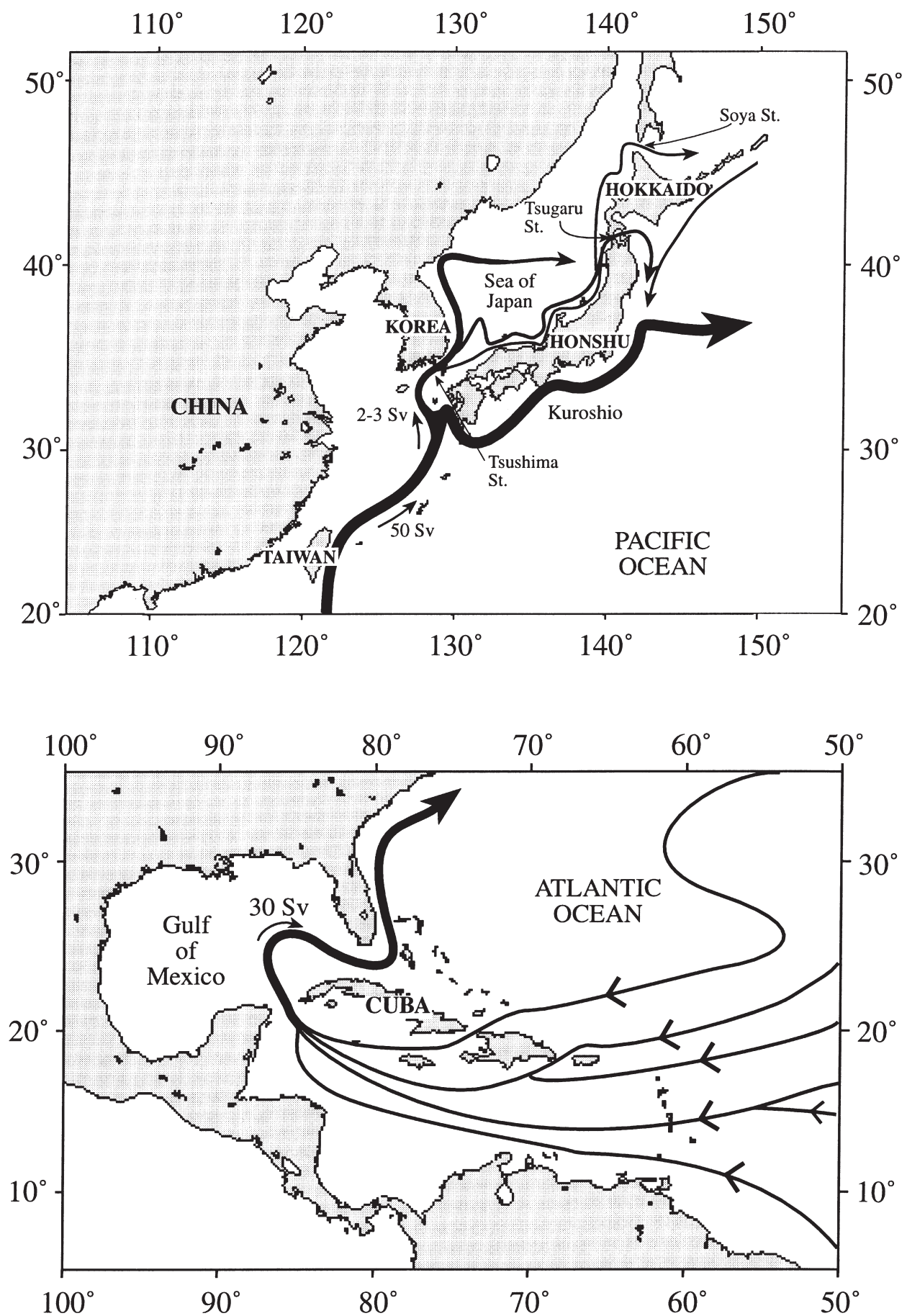

Fig. 1. The circulation pattern in the Japan Sea (top) and the Caribbean (bottom). On average, about 2-3 Sv enter the Sea of Japan and about $30 \mathrm{~Sv}$ enter the Caribbean. Adapted from Sugimoto (1990) and Schmitz (1996). 


\subsection{Classification}

By and large, there are two kinds of exchanges between oceans and marginal seas. The first is a density-controlled exchange and the second is a wind- or inertial-driven exchange. Examples of density-driven exchanges are the evaporative seas such as the Mediterranean, the Red Sea, and the Gulf of Hormuz. They are responsible for the formation of dense water and usually have an opposing two-layer exchange within the passages connecting them to the adjacent oceanic basins. Non-evaporative seas (e.g., the Caribbean and the Sea of Japan) usually display a one-directional baroclinic or barotropic exchange through the connecting passages. They can be divided into two subcategories, those with multiple passages connecting them to the large basins (e.g., the Caribbean) and those with a single connecting gap (e.g., the South China Sea). We shall focus here on marginal seas with multiple gaps; seas with a single gap will not be dealt with.

A distinction also needs to be made between marginal seas situated along the eastern boundary of wind-driven oceanic basins and those placed next to western boundaries. Since the eastern parts of the oceans are relatively calm compared to their western counterparts where intense boundary currents are present, it is expected that eastern marginal seas will be subject to less transport penetration than are western marginal seas. Ironically, however, there are really no (non-evaporative) eastern marginal seas to speak of. The Indonesian Seas are situated to the east of the Indian Ocean, but they are also connected to the western Pacific so that they belong to a 'mixed type' which is a different category altogether.

We shall focus our attention on non-evaporative seas connected to the basins with at least two gaps.

\subsection{Background}

For a review of general aspects of flows through straits and gaps, the reader is referred to Pratt (1990), which is a collection of investigations as well as review articles. The reader is also referred to Minato and Kimura (1980), Kawabe (1982a,b), Yoon (1982a,b,c), Sekine (1986, 1988), Wajsowicz (1993, 1994, 1996), Kim and Yoon (1996), Preller and Hogan (1998) and Hogan and Hurlburt (2000) for discussions on the penetration of water into marginal seas in general and into the Sea of Japan in particular. Reference is also made to Hurlburt and Thompson (1980) who deal with the throughflow in a marginal sea connected to the ocean via two passages.

The best estimate for the total flow through the Caribbean Sea is based on Richardson, Schmitz and Niiler (1969) measurements of the flow in the Florida Straits which give about 30 Sv. For the Sea of Japan, both Sugimoto (1990) and Toba, Tomizawa, Kurasawa and Hanawa (1982) suggest that the flow entering the Sea through the Tsushima Strait is much smaller, roughly 2-3 Sv. Although both the Tsushima Strait and the main exit straits, the Tsugaru and the Soya straits, are considerably smaller than the passages connecting the Caribbean to the Atlantic, they are not small enough to restrict the flow to the degree that they do.

For example, the Tsushima Strait is about $100 \mathrm{~m}$ deep and $200 \mathrm{~km}$ broad. With 
speeds comparable to those in the Florida Strait $\left(1-2 \mathrm{~ms}^{-1}\right)$ it could be transferring 20-40 Sv instead of the observed 2-3 Sv. Similarly, the Tsugaru Strait is $20 \mathrm{~km}$ wide and $130 \mathrm{~m}$ deep (at the sill) and could, therefore, accommodate transport of 3-6 Sv instead of the observed average of $<2 \mathrm{~Sv}$. Likewise, the Soya Strait is about $40 \mathrm{~km}$ broad and $70 \mathrm{~m}$ deep (at the sill) and could accommodate as much as 3-6 $\mathrm{Sv}$ instead of the average of half a Sverdrup. Overall, it seems that the Sea of Japan could have a much greater throughflow of, say, $6-12 \mathrm{~Sv}$ which is a few times larger than the observed throughflow (2-3 Sv).

A related aspect that needs to be examined is the effect of local winds over the marginal seas. In particular, it is important to examine the question whether the northeasterly trade winds over the Caribbean, which are much stronger than the winds over the Sea of Japan, can attribute to the difference in the transport. Although there is no doubt that the local winds affect the local flow, a simple scaling shows that, since the areas of both marginal seas are very small compared to their adjacent oceans (which are responsible for the generation of the western boundary currents), the effects of local winds on the transport is relatively small. Consequently, this effect is neglected to first order.

We should also point out that, although the surface circulation of the western Atlantic contains the meridional overturning cell (MOC) which does not have a counterpart in the Pacific, the total western boundary currents (WBC) transport in the two oceans is approximately the same. This is because of the much larger area of the Pacific which (through the wind field) compensates for the fact that the Pacific does not have an MOC. In view of this, the presence of the MOC in the Atlantic does not explain the much greater transport in the Caribbean compared to that of the Sea of Japan.

On the basis of the above, we shall examine the idea that the size and depth of the gaps as well as the local winds and the MOC are not the controlling elements in the Pacific-Sea of Japan exchange process. Specifically, we shall look at the possibility that it is the latitude of the Sea of Japan and its proximity to the Kuroshio separation latitude that restrict the flow into it. We shall show that the Sea of Japan throughflow would have been considerably greater had it been located farther to the south. This aspect was already briefly touched upon in Nof (1993), who showed that the transport of a ' $\beta$-controlled' penetration is given by the formula $\frac{g^{\prime} H^{2}}{2 f_{0}}\left(\frac{\beta L_{\mathrm{s}}}{f_{0}}\right)$, where $H$ is the western boundary current (WBC) undisturbed thickness offshore, $g$ ' its 'reduced gravity,' $f_{0}$ the mean latitude of the gap, $\beta$ the familiar linear variation of the Coriolis parameter with latitude, and $L_{\mathrm{s}}$ is the distance between the gap and the WBC separation latitude (with the gaps connecting the ocean and the marginal sea closed). This transport formula shows that the penetration decreases with increasing latitude. It breaks down, however, for gaps situated a distance of the order of the basin scale away from the WBC separation latitude because it neglects frictional processes within the WBC. Consequently, in the form given above, it cannot be applied to marginal seas such as the Caribbean.

It is important to realize that the above meridional transport within the marginal 
sea is driven by the zonal sea-level difference between the western boundary of the Pacific Ocean and the western edge of the penetrating current (in the marginal sea). As this hypothetical inviscid current is not returned to the modeled Pacific Ocean, there need not be any significant meridional sea-level difference along it. However, when the penetrating current is returned to the Pacific as is the case in our numerical experiments and in the model of Minato and Kimura (1980) (hereafter referred to as $\mathrm{MK}$ ), then the zonal sea-level difference across the marginal sea is generated by a meridional sea-level drop along the western boundary of the ocean. The two sealevel differences (i.e., the zonal and meridional differences) are identical in the case of a relatively short (wind-free) marginal sea connected to the ocean via inviscid flows through the channels. (By 'short' we mean that the meridional extent of the marginal sea is small compared to the meridional extent of the ocean.) Frictional forces within the marginal sea reduce the zonal sea-level difference (compared to the meridional sea-level drop) and thus reduce the transport through the marginal sea. We shall see that, in all the four baroclinic models that we shall be using, the frictional forces are small so that the zonal and meridional sea-level differences are practically identical.

The present study is an in-depth investigation of the (marginal seas) throughflow dependency on latitude. We shall use numerical simulations, Godfrey's island rule (Godfrey, 1989), Nof's general gap formula (Nof, 1995) and the MK formula to estimate the latitude dependency.

\subsection{Present approach}

Consider the idealized conceptual ocean-marginal sea system shown in Fig. 2. The oceanic basin is subject to an idealized zonal wind action and the marginal sea is 'wind-free.' Under such conditions (of a stress-free marginal sea) the changes that would occur as we gradually move the marginal sea northward are not caused by changes in the wind directly above the marginal sea but rather by changes in the marginal sea-ocean exchange process which we are interested in.

Our modelling efforts proceed as follows. First, the gaps are closed and we let the wind blow until a steady state is reached. (This state is referred to as the initial state.) We then open the gaps and let the model again reach a steady state. It is this second steady state that we are interested in.

We shall use four different baroclinic models to compute the throughflow, $T$. The first is to employ a layer-and-a-half isopycnic model. Second is to take the wall AB to be an island and use Godfrey's island rule to determine $T$ analytically from the wind stress along BC and AD (Section 3). The third is to apply Nof's (1993) ' $\beta$ controlled' formula to the gap (Section 4), and the fourth will use Nof's (1995) general gap formula (Section 5). As a supplement to these baroclinic models, we shall also apply MK's barotropic formula to the gaps (Section 6). Finally, we shall compare the outputs of all of the five models and show that, according to all four baroclinic models, the reason for the small penetration of Pacific water into the Sea of Japan is its relatively high latitude and not the size of the passages connecting it to the Pacific (Section 7). The barotropic model also shows a decrease of transport 


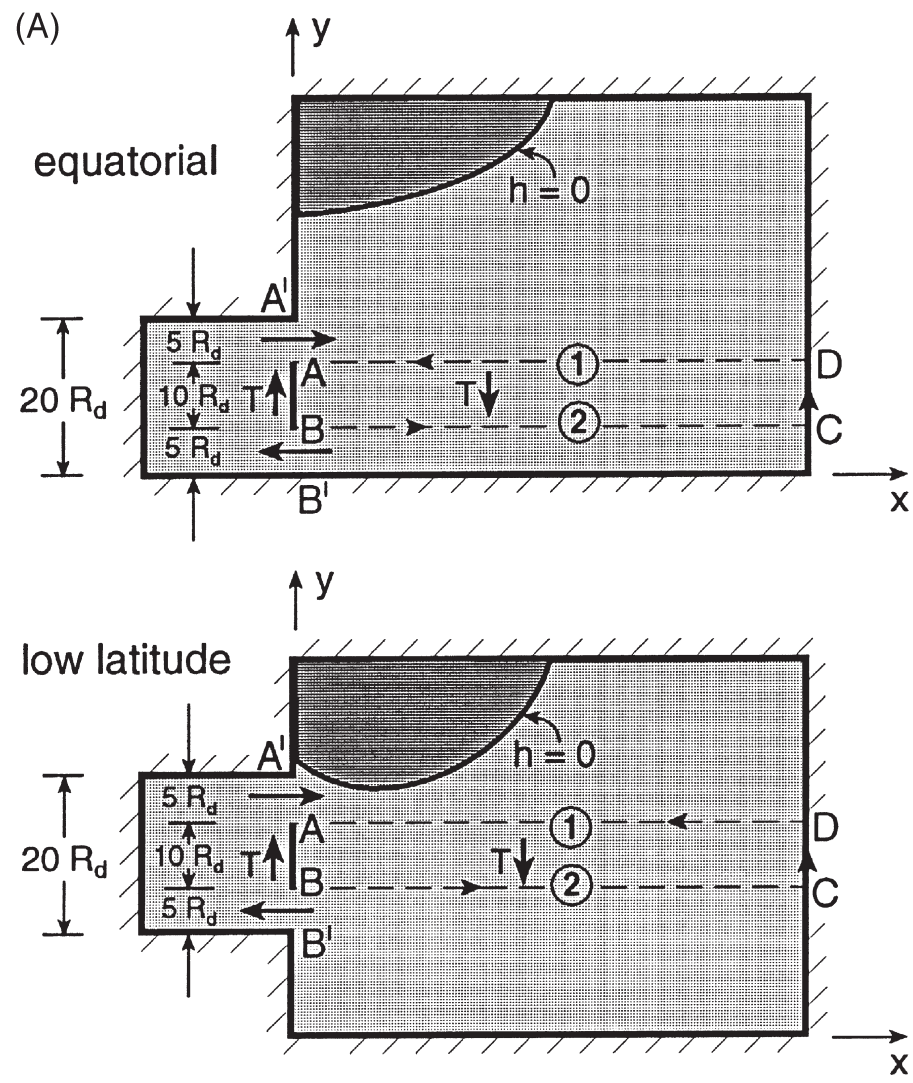

$A_{N}$
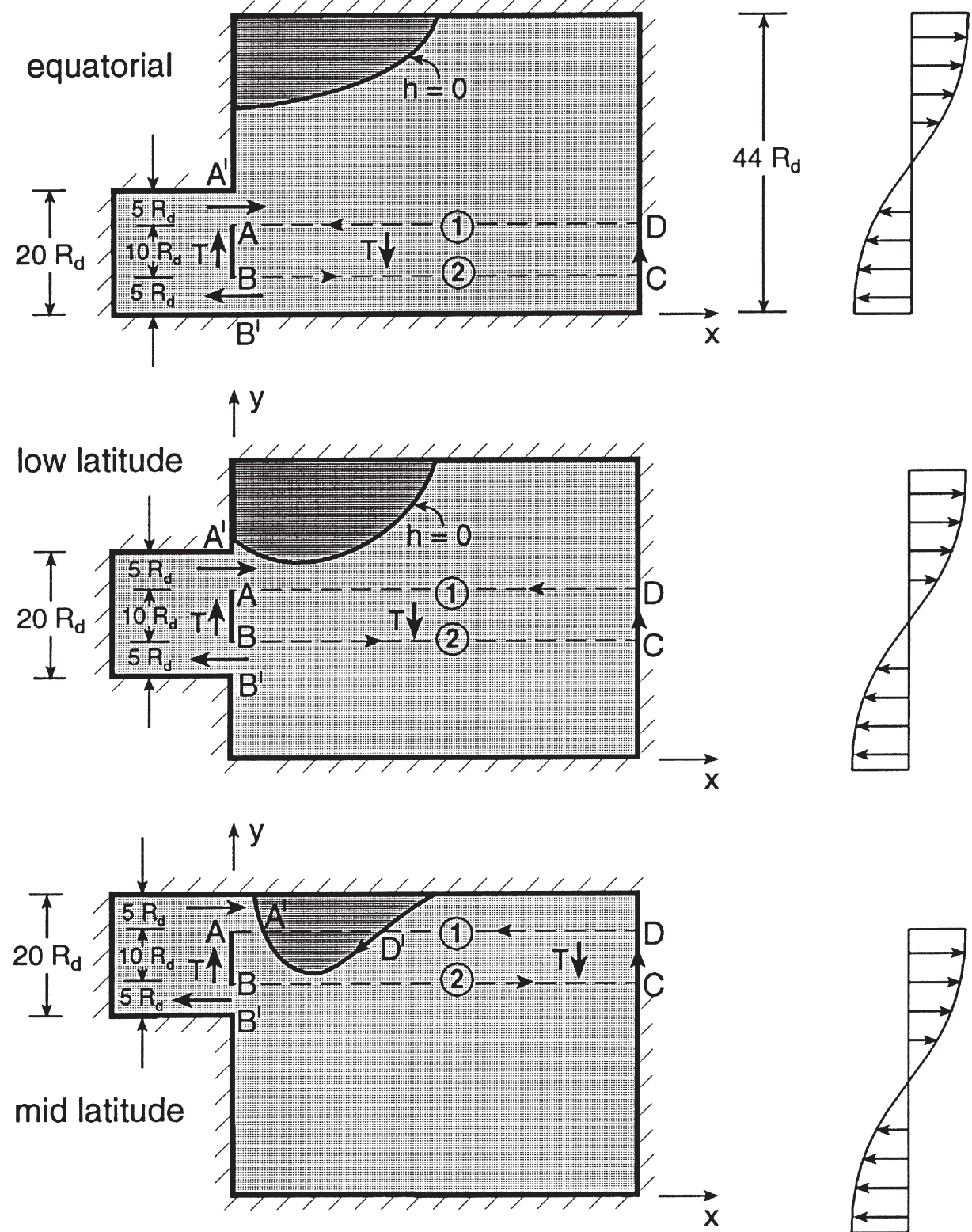

$\longrightarrow 10 R_{d} \longleftarrow 20 R_{d}$

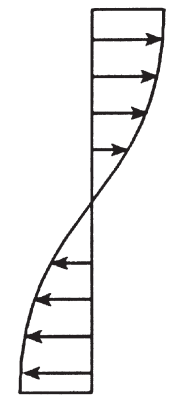

wind stress 
(B)

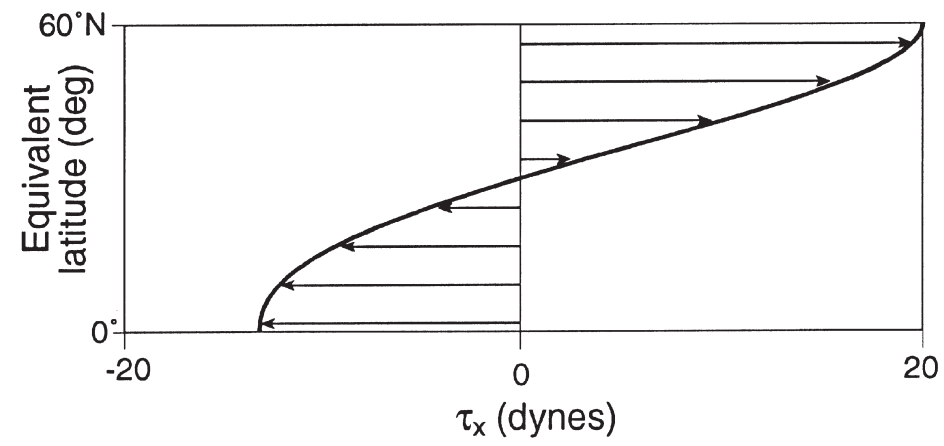

(C)

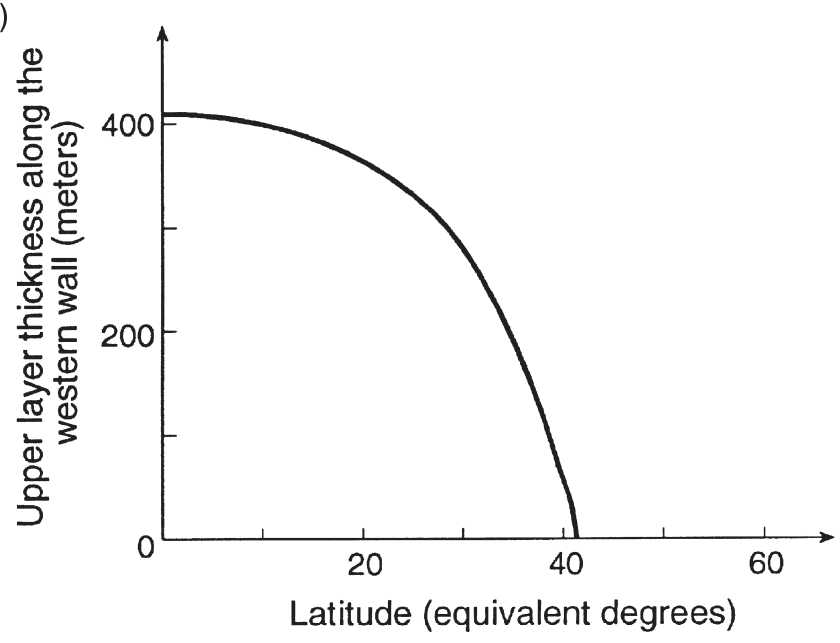

Fig. 2. (A) Opposite. Schematic diagram of the marginal sea model under study. To examine the sensitivity of the throughflow to the latitude of the sea we gradually vary the latitude of the marginal sea from equatorial (top) to low (central) and mid latitude (bottom). The idealized wind stress that we used is shown schematically on the right; its detailed structure is shown in (b). The throughflow $T$ is computed using four different baroclinic models and a barotropic model (see text). In all five cases the marginal sea is not subject to any wind stress. The central panel corresponds to the Caribbean (whose mean latitude is roughly $22^{\circ} \mathrm{N}$ ) and the bottom panel to the Sea of Japan (whose mean latitude is about $42^{\circ} \mathrm{N}$ ). Note that, in the cases where the separation line $(h=0)$ extends south of the island northern tip (lower panel), the (counterclockwise) integration path (leading to Godfrey's island rule) has been modified so that the integration is not performed over the passive lower layer. Also, note that, for clarity, the figure is not drawn to scale. (B) The idealized (sinusoidal) wind field used in the study (adapted from Hellerman \& Rosenstein, 1983). The 'equivalent latitude' is not the actual latitude used in the runs (which was suppressed by the magnification of $\beta$ ) but rather the latitude corresponding to the 'natural' unmagnified value of $\beta\left(2 \times 10^{-11} \mathrm{~m}^{-1} \mathrm{~s}^{-1}\right)$. (C) The initial upper layer thickness along the western wall. In this state the gaps are closed and there is no light water in the marginal sea. Note that separation occurs around $42^{\circ} \mathrm{N}$ (D) On following page. The Bernoulli function (upper panel) and its components (lower panel) along the western wall of the oceanic basin during the initial steady state (i.e., the gaps are closed and there is no upper layer water in the marginal sea). Note that, as expected, the Bernoulli is not conserved as one moves across the entire basin (i.e., from equatorial to mid-latitude). However, if one moves merely a few degrees it is approximately constant. 

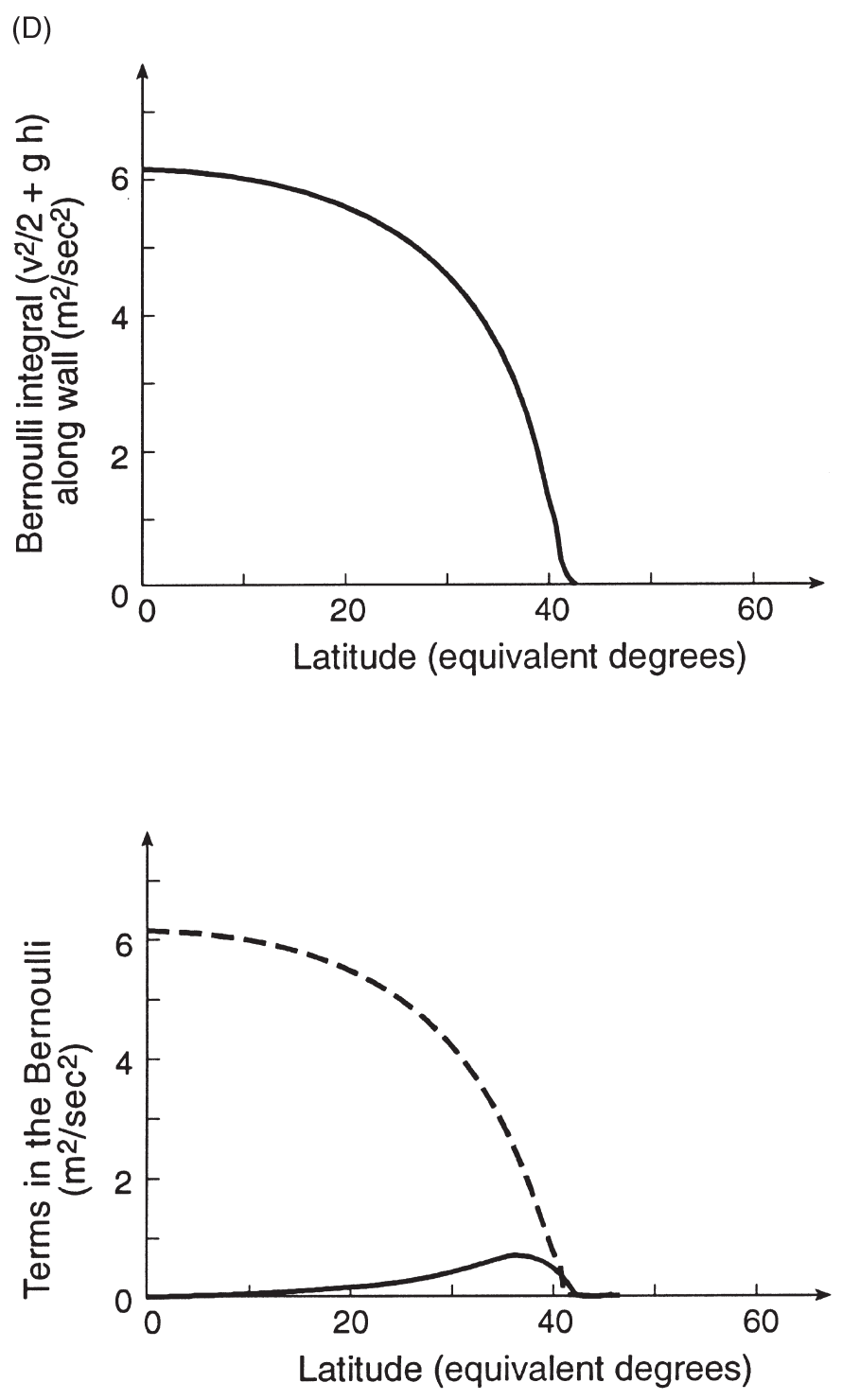

Fig. 2. (continued)

with latitude but this occurs at a much higher latitude than that corresponding to the Sea of Japan.

\section{Numerical simulations}

As already mentioned, to examine the ocean-marginal sea exchange process we first consider a 'reduced gravity' rectangular basin with an attached rectangular mar- 
ginal sea (Fig. 2). We shall vary the latitude of the marginal sea, measure the throughflow, and then plot the computed transport versus the latitude of the marginal sea.

\subsection{The numerical model}

We use the reduced gravity version of the model developed by Bleck and Boudra (1981, 1986) and improved by Bleck and Smith (1990). The advantage of this model is its use of the 'Flux Corrected transport' algorithm (Boris \& Book, 1973; Zalesak, 1979) for the solution of the continuity equation. This algorithm employs a higher order correction to the depth calculations and allows the layers to outcrop and stay positive definite. The resulting scheme is virtually non-diffusive and conserves mass. For these reasons, the model is the most suitable for our problem. The active layer is an enclosed feature, while the rest of the layer has zero depth.

The modeled basin is rectangular extending from the equator to $60^{\circ} \mathrm{N}$. The equations of motion are the two (familiar) momentum equations,

$$
\begin{aligned}
& \frac{\partial u}{\partial t}+u \frac{\partial u}{\partial x}+u \frac{\partial u}{\partial y}-\left(f_{0}+\beta y\right) v=-g^{\prime} \frac{\partial h}{\partial x}+\frac{v}{h} \nabla \cdot(h \nabla u)+\frac{\tau^{\mathrm{x}}}{\rho h}-k u \\
& \frac{\partial v}{\partial t}+v \frac{\partial v}{\partial x}+v \frac{\partial v}{\partial y}+\left(f_{0}+\beta y\right) u=-g^{\prime} \frac{\partial h}{\partial y}+\frac{v}{h} \nabla \cdot(h \nabla v)+\frac{\tau^{\mathrm{y}}}{\rho h}-k v,
\end{aligned}
$$

and the continuity equation,

$$
\frac{\partial h}{\partial t}+\frac{\partial(h u)}{\partial x}+\frac{\partial(h v)}{\partial y}=0
$$

where the conventional notation is given in the Nomenclature (e.g., $u$ and $v$ are the horizontal velocity components, $h$ is the upper layer thickness, $v$ is the viscosity, $\tau^{\times}$ and $\tau^{y}$ are the wind stress components, $\rho$ is the density, and $k$ is the interfacial friction coefficient).

The model uses the Arakawa (1966) C-grid. The $u$-velocity points are shifted onehalf grid step to the left from the $h$ points, the $v$-velocity points are shifted one-half grid step down from the $h$ points, and the vorticity points are shifted one-half grid step down from the $u$-velocity points. This grid allows for reducing the order of the errors in the numerical scheme. The grid scale is $15 \mathrm{~km}$, and the solution is advanced in time using the leap-frog scheme with a time step of $540 \mathrm{~s}$. The velocity fields are smoothed in time in order to stabilize the numerical procedure. The velocities for the weightless grid points are set to zero.

To make our runs more economical, we artificially magnified both $\beta$ and the wind stress (by a factor of five and ten, respectively) and artificially reduced both the meridional and zonal basin scales (again by a factor of five and ten, respectively). With these changes, our $\beta$ was $11.5 \times 10^{-11} \mathrm{~m}^{-1} \mathrm{~s}^{-1}$, the zonal basin size was 600 $\mathrm{km}$, the meridional basin size was $1320 \mathrm{~km}$, and the Coriolis parameter remained unaltered (for the new latitudes). The linear drag coefficient, $k$, was also increased by a factor of 10 (to $1 \times 10^{-6} \mathrm{~s}^{-1}$ ) so that the increased wind stress is balanced. With a grid size of $15 \mathrm{~km}$, an (unaltered) reduced gravity of $1.5 \times 10^{-2} \mathrm{~m} \mathrm{~s}^{-2}$ and an undis- 
turbed depth of $300 \mathrm{~m}$ (giving a Rossby radius of $30 \mathrm{~km}$ ), each of our computational runs lasted for about 40 minutes instead of at least a week each run would have lasted had we not modified $\beta$, the wind stress, and the basin size.

The downside of the above modifications is an unnaturally large ratio between the eddies' size (the Rossby radius) and the basin's size. As we shall shortly see, this is not really a difficulty in our runs because in our case the eddies do not play a major role in the mass exchange process. Furthermore, this unnatural eddy-basinratio issue can be easily resolved by using a very large horizontal eddy viscosity coefficient $\left(16,000 \mathrm{~m}^{2} \mathrm{~s}^{-1}\right)$ that eliminates any long-lived eddies such as Gulf Stream rings. This essentially implies that our model is not an eddy-resolving model. The (apparent) large eddy coefficient is in fact not so large because of the magnified value of $\beta$ which keeps the frictional Munk layer relatively thin $\left[(v / \beta)^{1 / 3} \sim 25 \mathrm{~km}\right]$. The simplified wind stress profile that we used is shown in Fig. $2 b$.

We performed a total of seventeen experiments with marginal seas of a fixed size and gradually varying latitudes. Our gaps were five times the Rossby radius, the zonal extent of the sea was ten times the Rossby radius, and the meridional extent was twenty times the Rossby radius. With these choices the wall-island length was ten times the Rossby radius. The initial condition for all of our experiments was that the gaps were closed and the marginal sea did not contain any light water. The corresponding thickness and Bernoulli function along the western wall are shown in Fig. 2c and d. We begin our simulations by aligning the southern wall of the marginal sea with the southern boundary of the basin(the equator). This experiment is referred to as Run 1. We then gradually increased the mean latitude of the sea by increments of $1.5 R_{\mathrm{d}}$ until the northern wall of the sea was aligned with the northern wall of the basin (Run 17).

\subsection{Results}

Typical results are shown in Figs. 3 and 4 which display the (steady) upper layer transport and thickness contours. We see from Fig. 3 that as we move from equatorial to low latitudes the transport that penetrates into the marginal sea increases. This is because the southwest corner of the basin is relatively quiet and, consequently, the sea-level difference between the entering and exiting gaps is very small. In other words, since in these cases there is no WBC adjacent to the island, there is almost no frictional pressure drop along the western boundary of the basin and the associated zonal sea-level difference across the marginal sea is also small. As a result, not much fluid penetrates into the marginal sea when the southern gap is close to the southern wall. As the gap's latitude increases, the region next to the gap becomes more dynamic, the sea-level difference between the two gaps increases (because of the frictional pressure drop created by the WBC), and, consequently, the zonal pressure gradient in the marginal sea increases and more water penetrates into the marginal sea.

We see from Fig. 4 that the (penetration) transport increase with increasing gap latitudes does not hold for all latitudes. As we move from low to mid latitudes the reverse is true; i.e., the penetration transport decreases with increasing gap latitude. 
run 1
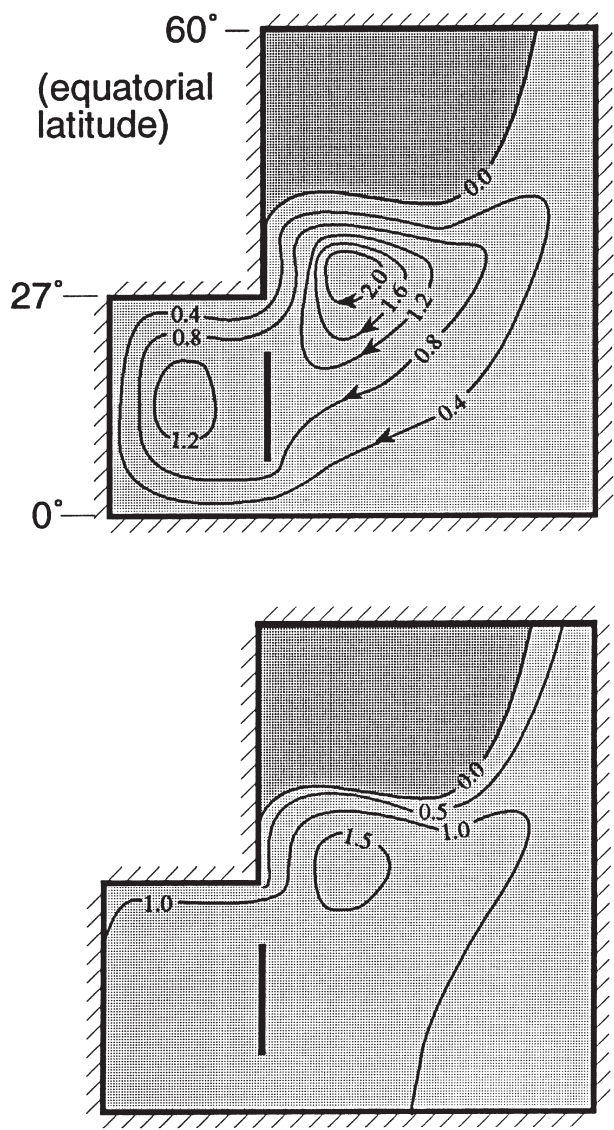

run 5
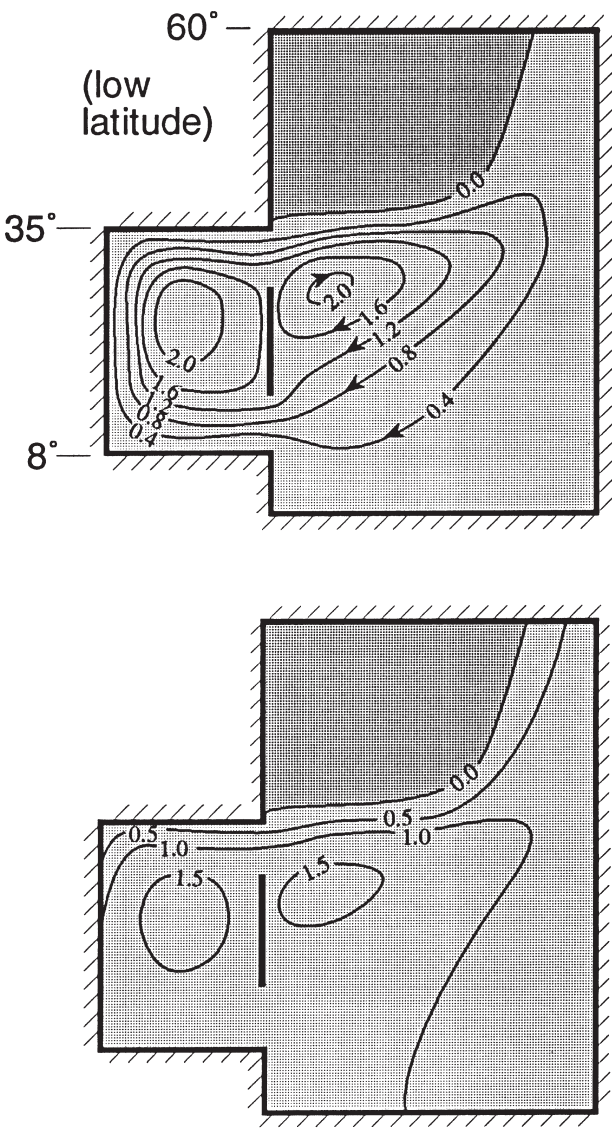

Fig. 3. Nondimensional transport contours (upper panels) and thickness contours (lower panels) for two simulations, one for an equatorial marginal sea (run 1, left panels) and one for low-latitude marginal seas (run 5, right panels). The transport is nondimensionalized by $g^{\prime} H^{2} / 2 f_{0}$ (with $g^{\prime}=0.015 \mathrm{~m} \mathrm{~s}^{-2}, H=300 \mathrm{~m}$ and $\left.f_{0}=6.8 \times 10^{-5} \mathrm{~s}^{-1}\right)$ and the thickness with the undisturbed depth $(200 \mathrm{~m})$. The Rossby radius is $30 \mathrm{~km}$. To eliminate eddies (see text) we used a high viscosity of $16,000 \mathrm{~m}^{2} \mathrm{~s}^{-1}$. Our magnified $\beta\left(11.5 \times 10^{-11}\right.$ $\mathrm{m}^{-1} \mathrm{~s}^{-1}$ ) results in a Munk layer thickness of $25 \mathrm{~km}$. Run 1 corresponds to a marginal sea whose southern boundary coincides with the equator whereas run 5 corresponds to a low latitude marginal sea. Note that in run 1 there are two western boundary currents (WBC), one in the main basin(north of the island) and one in the marginal sea. Run 5, on the other hand, has only one WBC (in the marginal sea). It has, however, two strong zonal boundary currents, one along the northern and another along the southern boundaries of the marginal sea. Clearly, more water enters the low latitude marginal sea (run 5) than the equatorial marginal sea (run 1) because the gaps are situated in a calm region in run 1 and in an active region in run 5 . Shaded regions denote areas where there is no upper layer. 
run 11
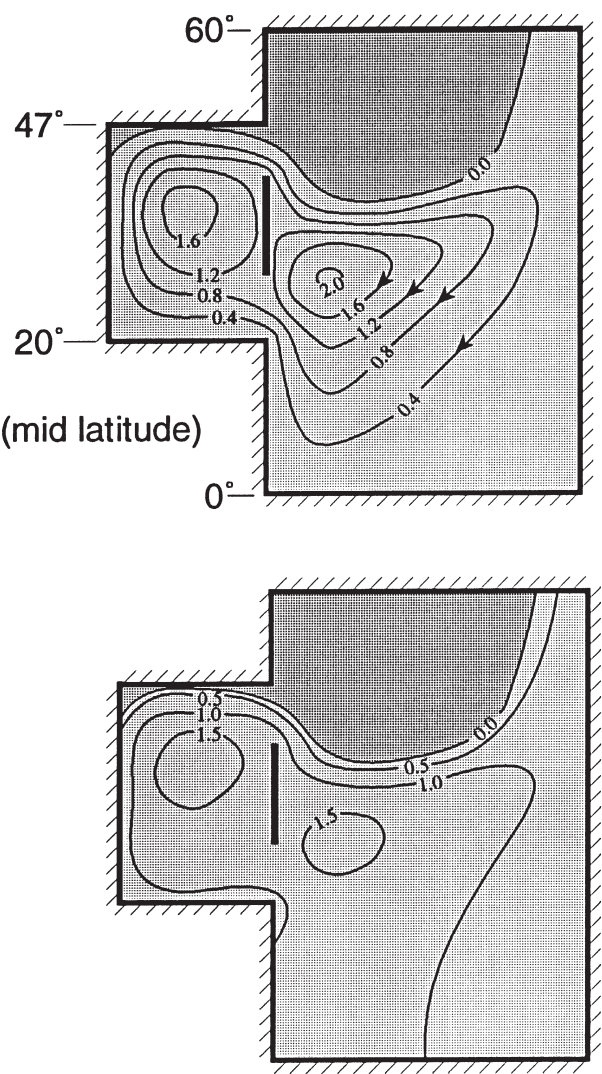

run 15
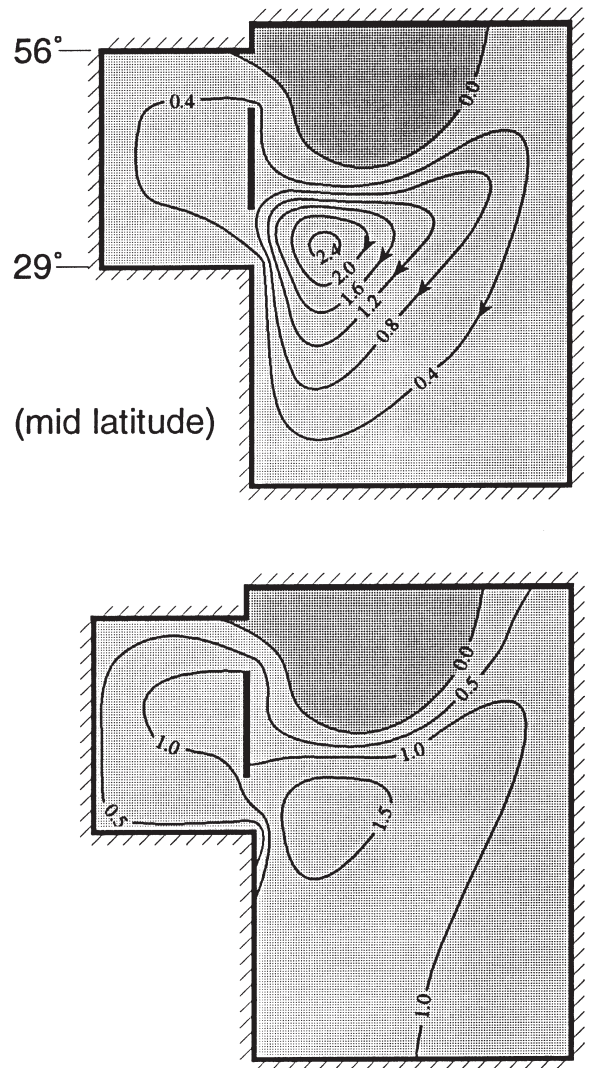

Fig. 4. The same as Fig. 3 except that we show here two mid latitude seas; one (run 11) has a latitude lower than the other (run 15). In contrast to the equatorial and low latitude seas where the transport increased with increasing latitude, the transport here decreases with increasing latitude because of the decreasing WBC near-wall thickness in the basin (see text).

This is because of the decrease in the WBC near wall thickness which results from the increase in the Coriolis parameter and the interface slope. This decrease also implies a decrease in the sea-level difference between the two gaps (and a resulting decrease in the corresponding zonal pressure gradient within the marginal sea) because the northeast part of the marginal sea is situated northward of the WBC separation so that the sea level there is almost fixed. The dependence of the penetration transport on the gap's latitude is shown in Fig. 5 which also displays the analytical calculations according to the island rule (Godfrey, 1989), the ' $\beta$-controlled' formula (Nof, 1993), the general gap formula (Nof, 1995) and the barotropic MK solution. These calculations will be discussed in detail in the next three sections.

Two points should be added before proceeding to the detailed presentation of the analytical calculations. First, note that the wind-free marginal sea displays enclosed 


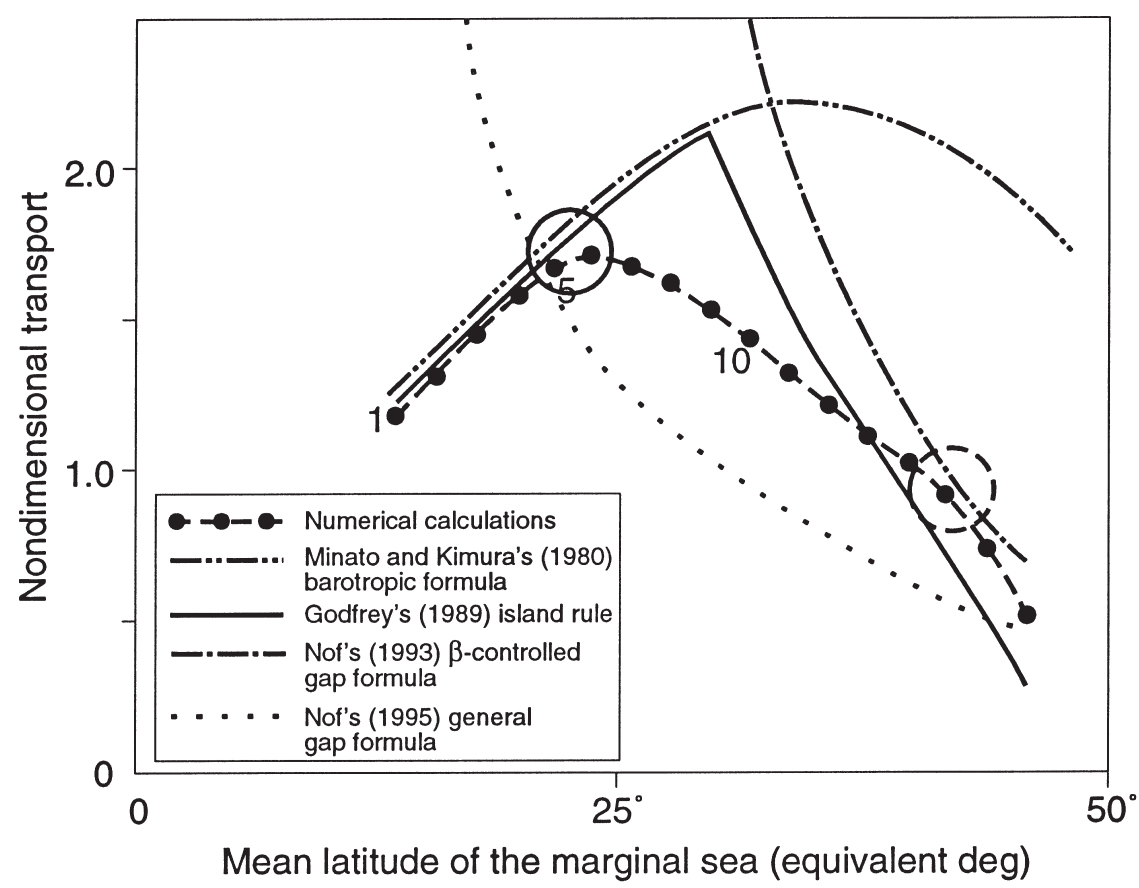

Fig. 5. An inter-comparison of the numerically simulated baroclinic transport within the marginal sea (dashed line), the baroclinic transport calculated with Godfrey's island rule (solid line), the baroclinic transport calculated from Nof's (1993) $\beta$-controlled gap formula (dashed-dotted line), Nof's (1995) general gap formula (dotted line) and the (barotropic) transport according to Minato and Kimura (dasheddouble-dotted line). All are shown as a function of the sea's mean latitude. Numerals indicate the number of the numerical experiment. Solid open circle corresponds to the Caribbean (mean equivalent latitude of approximately $22^{\circ} \mathrm{N}$ ) and dashed circle corresponds to the Sea of Japan (mean latitude of approximately $41^{\circ} \mathrm{N}$ ). Note that the high latitude numerical deviations from the island rule calculations are due to the position of the separated WBC which flows eastward. In these cases the zonal jet is situated south of the island's northern tip so that the integration path is cutting through it. Under such conditions, both the neglected frictional terms and the nonlinear terms are no longer small. Also note that the $\beta$-controlled gap formula gives the correct results where it is supposed to (i.e., when the gap is in the vicinity of the WBC separation). This is also the case with the general gap formula. The deviation between Minato and Kimura's barotropic formula and the numerics is high in mid latitudes due to the surfacing of the interface.

circulation contours because the horizontal friction transfers momentum from the surrounding flow to the core. Second, note that Run 15 displays stronger circulation than Run 11 because, as the marginal sea is moved from low to mid latitude, it holds less and less upper layer water so that the mean thickness (and hence the transport) in the main basin increases.

\section{Island rule calculations}

We shall use a slightly modified version of the classical island rule which allows for a surfacing upper layer. The extension of the original rule to this case is straight- 
forward but a bit tricky because the limit $h \rightarrow 0$ corresponds to a wind stress which must be taken to be zero even though it is actually finite. To understand this modified island rule calculation clearly and to compare it with the numerics, it is best to derive the modified rule from first principles. This is presented below.

\subsection{Governing equations}

Consider the model shown in Fig. 2a. Following Godfrey (1989), (see also Godfrey \& Masumoto, 1999), we shall use the familiar vertically integrated equations of motion for the upper layer,

$$
\begin{aligned}
& -f V=-\frac{1}{2} g^{\prime} \frac{\partial}{\partial x}\left(h^{2}\right)+\frac{\tau^{\mathrm{x}}}{\rho} \\
& f U=-\frac{1}{2} g^{\prime} \frac{\partial}{\partial y}\left(h^{2}\right)-R V \\
& U_{x}+V_{y}=0
\end{aligned}
$$

where, as before, the notation is conventional; i.e., $h$ is the upper layer thickness, $U$ and $V$ are the vertically integrated transports in the $x$ and $y$ direction (here, $x$ is pointing eastward and $y$ is pointing northward), $f$ is the Coriolis parameter (varying linearly with $y$ ), $g$ ' is the 'reduced gravity' $g \Delta \rho_{1} / \rho$ (where $\Delta \rho_{1}$ is the density difference between the upper and the intermediate layer and $\rho$ is the density of the upper layer), $\tau^{\mathrm{x}}$ is again the wind stress in the $x$ direction, and $R$ is an interfacial friction coefficient which need not be specified for the purpose of our analysis. The thick lower layer velocities are taken to be, on average, small compared to those of the upper layer.

Elimination of the pressure terms between Eqs. (4) and (5) and consideration of Eq. (6) gives

$$
\beta V=-\frac{1}{\rho} \frac{\partial \tau^{\mathrm{x}}}{\partial y}-R \frac{\partial V}{\partial x},
$$

which, for the inviscid ocean interior, reduces to the familiar Sverdrup relationship,

$$
\beta V=-\frac{1}{\rho} \frac{\partial \tau^{\mathrm{x}}}{\partial y} .
$$

Four comments should be made with regard to Eqs. (4)-(8). First, in this model, energy is supplied by the wind over the entire ocean and is dissipated through interfacial friction [i.e., the RU term in Eq. (5)] within the limits of the WBC system. This interfacial friction is not present in Eq. (4) because it is assumed that RU is small and negligible. Furthermore, since the velocities are small in the ocean interior the frictional term is negligible there. The absence of RU from Eq. (4) implies that the model cannot effectively handle zonal boundary currents such as a separated WBC because these currents correspond to a large $U$. We shall see later that this is an 
important weakness in the application of the island rule to our case, and explains the discrepancy between the numerical calculations and the island rule calculations for mid-latitude seas.

Second, Eq. (4) holds both in the sluggish interior away from the boundaries and in the intense WBC where the flow is geostrophic in the cross-stream direction. Within the WBC the balance is between the Coriolis and pressure terms with the wind stress playing a secondary role. In the interior, on the other hand, the velocities are small and, consequently, all three terms are of the same order. Third, the inertial terms are excluded from the model but, to allow the interface to surface, nonlinearity is included in the pressure terms (via the square of the thickness). Since the model does not include relative vorticity within the WBC, it implicitly assumes that all the relative vorticity generated by moving the fluid meridionally (within the boundary layer) is dissipated immediately after its creation.

Fourth, in this model, the frictional coefficient determines the width of the (unseparated) WBC but not its length (which, in turn, is controlled by the wind field and the thickness along the eastern boundary). In other words, the WBC adjusts its width in such a way that the total energy input (by the wind) is always dissipated along its base.

Finally, it is perhaps appropriate to comment on the assumption of an inert lower layer because this assumption is frequently misunderstood. In contrast to common statements, the assumption does not imply negligible transports in the lower layer. It merely implies that the horizontal pressure gradients in the thick lower layer are small compared to the horizontal pressure gradients in the upper layer. This means that, on average, the speeds in the lower layer are small compared to the speeds in the upper layer; since the lower layer thickness is much greater than the upper layer thickness, the transports in the intermediate layer can be of the same order as those of the upper layer. Furthermore, since Ekman flows do not necessarily involve horizontal pressure gradients, they are not necessarily excluded from the intermediate layer.

\subsection{Boundary conditions}

The boundary condition along the solid boundaries is, of course, the familiar nonormal flow into the western and eastern walls. Also, since friction is taken to be unimportant in the ocean interior, for an eastern boundary Eq. (5) is modified to

$$
f U=-\frac{g^{\prime}}{2} \frac{\partial}{\partial y}\left(h^{2}\right)
$$

Since $U \equiv 0$ along both the eastern boundary of the ocean basin and the eastern boundary of the marginal sea, it follows immediately from Eq. (9) that

$$
h_{\mathrm{D}}=h_{\mathrm{C}} \text { and } h_{\mathrm{A}}=h_{\mathrm{B}}
$$

where $h_{\mathrm{A}}, h_{\mathrm{B}}, h_{\mathrm{C}}$, and $h_{\mathrm{D}}$ are the upper layer thicknesses at $\mathrm{A}, \mathrm{B}, \mathrm{C}$, and D, respectively (Fig. 2). As mentioned, along the western boundary the zonal velocity compo- 
nent also vanishes. However, in contrast to the eastern boundary, the thickness is not uniform along this boundary because of the frictional term.

\subsection{Integration of the momentum equation}

To derive the transport formula for the flow around the island, we integrate Eq. (4) from the western to the eastern boundary along the latitudes connecting the tip of the island to the eastern boundary (i.e., sections 1 and 2 shown in Fig. 2),

$$
\begin{aligned}
& -f_{1} T=\frac{g^{\prime}}{2} \int_{A}^{D} \frac{\partial}{\partial x}\left(h^{2}\right) \mathrm{d} x-\int_{A}^{D}\left(\frac{\tau^{\mathrm{x}}}{\rho}\right) \mathrm{d} x \\
& -f_{2} T=\frac{g^{\prime}}{2} \int_{B}^{C} \frac{\partial}{\partial x}\left(h^{2}\right) \mathrm{d} x-\int_{B}^{C}\left(\frac{\tau^{\mathrm{x}}}{\rho}\right) \mathrm{d} x,
\end{aligned}
$$

where $T$ is the net meridional transport across the ocean and $f_{1}$ and $f_{2}$ are the Coriolis parameters along sections 1 and 2 . In contrast to the classical closed basin approach which requires the WBC transport to be equal and opposite to the interior Sverdrup transport so that there is no net meridional transport (i.e., $T=0$ ), we do not require the two transports to have any particular relationship. For reasons that will become momentarily apparent, Eqs. (11a) and (11b) are now rewritten in the form,

$$
\begin{aligned}
& -f_{1} T=\frac{g^{\prime}}{2}\left(h_{\mathrm{D}}^{2}-h_{\mathrm{A}}^{2}\right)-\int_{A}^{D}\left(\frac{\tau^{\mathrm{x}}}{\rho}\right) \mathrm{d} x \\
& -f_{2} T=\frac{g^{\prime}}{2}\left(h_{\mathrm{C}}^{2}-h_{\mathrm{B}}^{2}\right)-\int_{B}^{C}\left(\frac{\tau^{\mathrm{x}}}{\rho}\right) \mathrm{d} x .
\end{aligned}
$$

By subtracting Eq. (12b) from Eq. (12a) and taking into account the eastern boundary conditions Eq. (10), we find the desired Godfrey's formula,

$$
T=\frac{-1}{f_{1}-f_{2}}\left[\int_{B}^{C} \frac{\tau^{\mathrm{x}}}{\rho} \mathrm{d} x+\int_{D}^{A} \frac{\tau^{x}}{\rho} \mathrm{d} x\right],
$$

Note that the procedure leading to Eq. (13) is analogous to a counterclockwise integration of Eqs. (4)-(6) over the path $\mathrm{ABCD}$ which includes the island (i.e., the integration from $\mathrm{A}$ to $\mathrm{B}$ is done over the western side of the island rather than the eastern side of the island). Also, note that, in cases where the separation line (i.e., the zero thickness line) is situated south of the island's northern tip (i.e., south of 
section $\mathrm{AD}$ ), the integration path needs to be modified in the manner shown in the lower panel of Fig. 2. With the modified path, the integration along ABCDD'A'A is done only over the upper layer, as should be the case.

An important aspect of the integration along the zero thickness line segment $\left(\mathrm{D}^{\prime} \mathrm{A}^{\prime}\right)$ is that since, by definition, there is no transport across it and no pressure gradient along it, the wind stress along it must be taken to be zero as well. This is so, even though the actual wind stress along the zero thickness line is not zero. It is rationalized by the argument that in the analytical derivation of Eqs. (4)-(6) one assumes that both the stress along the bottom of the upper layer and the lateral friction is zero. When $h \rightarrow 0$, this assumption is no longer valid because, in this limit, either the bottom stress or the lateral friction must cancel the surface stress. This is the case because there is no pressure gradient, cross flow, or long-front nonlinearity to balance the imposed wind stress along $h=0$. Our numerical calculations show that it is the bottom stress rather than lateral friction which cancels the wind stress. (This can be easily verified by examining the cross front scales which are much too large to allow a significant lateral friction.)

Taking the wind stress to be zero along the front is satisfactory as far as the comparison between the analytics and the numerics is concerned but is not an entirely satisfactory scenario as far as the analytical model is concerned. This is because it begs the question of how close to the zero thickness line one can get and still take the bottom stress to be zero. At this stage, there is no satisfactory answer to this question, and we have no choice but to accept this difficulty as a possible weakness of the analytical model.

\subsection{Results}

The results of the island rule calculations are shown with the solid line in Fig. 5. We see that when the marginal sea is situated in equatorial or very low latitudes (up to simulation 5) the agreement between the island rule calculations and the numerics is excellent. However, the agreement decreases significantly as the latitude of the sea increases. This is because, as the mean latitude of the sea increases, the southern gap gets closer and closer to the latitude of the eastward flowing separated WBC. Ultimately, the marginal sea is situated so far north that the zonal separated WBC is situated south of the northern gap (see, e.g., Run 11 in Fig. 4). Under such conditions, the jet cuts through the integration path and, consequently, both the frictional forces in the northern segment of our integration (see section AD in the lower left panel of Fig. 4 and the nonlinear terms) are no longer small and cannot be neglected.

As a result of this unjustified neglect, the agreement between the island rule calculations and the numerical simulations is not as good as before though it is still pretty good. As expected, in this regime the simulated transport is less than the island rule transport reflecting the tendency of friction to slow down the fluid. Pedlosky, Pratt, Spall and Helfrich (1997) encountered similar weaknesses of the rule. 


\section{The ' $\beta$-controlled' gap formula}

The derivation of Nof's (1993) $\beta$-controlled penetration flux formula is somewhat tedious and need not be repeated here. It is sufficient to say at this stage that the calculations are based on the idea that, conceptually, the gaps can be envisioned as initially closed by imaginary gates. At $t=0$ the conceptual gates are removed and it is assumed that the transport reached after the completion of an initial adjustment period is equal to the ultimate steady transport through the gaps.

The final formula is simple and can be easily explained. It states that the flux through a mid-latitude gap in a meridional western boundary is,

$$
T=\frac{g^{\prime} H^{2}}{2 f_{0}}\left(\frac{\beta L_{\mathrm{s}}}{f_{0}}\right),
$$

where $H$ is the WBC undisturbed thickness offshore (i.e., the thickness along its eastern edge), $f_{0}$ is the Coriolis parameter of the gap, and $L_{\mathrm{s}}$ is the distance between the center of the entrance gap (i.e., the upstream southern gap) and the separation latitude of the WBC (taking place with the gaps closed). Note that, with this formulation, the northern gap is required to be situated north of the WBC separation (with the gaps closed). The offshore upper layer thickness can be found from Eqs. (4)(8) to be,

$$
H=\left(\frac{2}{g^{\prime}}\right)^{1 / 2}\left[\frac{f_{0}}{\beta \rho} \int_{0}^{L_{\mathrm{b}}} \frac{\partial \tau}{\partial y} \mathrm{~d} x-\int_{0}^{L_{\mathrm{b}}} \frac{\tau}{\rho} \mathrm{d} x\right]^{1 / 2},
$$

where $L_{\mathrm{b}}$ is the basin's length (see, e.g., Nof, 1995). It turns out that, in our case, this thickness is very close to the basin's undisturbed depth. (Note, however, that $H$ is not the thickness along the eastern boundary but rather the thickness several deformation radii offshore.)

Eq. (14) states that the transport is geostrophic and is based on the near wall thickness which is established when the gaps are closed. For short distances between the gap and the WBC separation, this thickness is simply $\left(\beta L_{\mathrm{s}} / f_{0}\right)^{1 / 2} H$ (see, e.g., Nof, 1993). In the derivation of Eq. (14) it has been assumed that the water penetrating into the marginal sea does not return to the basin but rather is removed from the basin altogether. Furthermore, it has been assumed that, since Kelvin waves require a wall on their right-hand side (looking downstream), no information can propagate from the entering gap to the separation point. As a result of these two assumptions, the position of the separation point is taken to be unaffected by the removal of the conceptual gates which initially block the gaps. We shall see in Section 5 that these two assumptions are not made in the derivation of the general gap formula.

The flux calculated using Eq. (14) is shown with the dashed-dotted line in Fig. 5. We see that, as long as the gap is fairly close to the WBC separation point (i.e., mid-latitude seas), the agreement is excellent. As the distance between the two increases (i.e., the latitude decreases), the discrepancy between the two calculations also increases. This is to be expected because the formula is based on an application 
of Bernoulli to the streamline connecting the gap and the WBC separation point. This application essentially assumes that the fluid does not suffer any energy loss as it moves from the gap to the separation point. In reality, there is, of course, an energy loss through interfacial friction along the base of the WBC. This loss is small for high-latitude gaps but (as shown in Fig. 5) increases dramatically with increasing distance between the gap and the WBC separation point (decreasing mean marginalsea latitude).

Note that, although $\beta$ is included in Eq. (15), the gap formula does not really involve $\beta$ directly because it is assumed that the gap's length is small compared to the basin's meridional extent. Under such conditions, $\beta$ is only important as far as the establishment of the WBC in the first place. The solution suffers, therefore, from the potentially serious weakness that it contains steady eastern boundary currents within the marginal sea. Clearly, such boundary currents cannot be maintained and, consequently, they would ultimately drift westward as a result of $\beta$. In our present calculation it is simply assumed that, although $\beta$ shifts the position of the current penetrating into the marginal sea from the eastern boundary of the marginal sea to its western boundary, it does not affect the transport of the current in the marginal sea. This is supported by the numerics.

\section{General gap formula}

The general gap formula (Nof, 1995) is somewhat less restrictive than the $\beta$ controlled formula which we just presented because it does not involve the assumption of an unaltered WBC separation. When this assumption regarding the unaltered WBC separation latitude (made in the derivation of Eq. (14)) is relaxed, one needs an additional constraint in order to close the problem. Nof's (1995) $f$-plane solution involves a 'front progression condition' (implying that $\frac{D}{D t}\left(v+f_{0} x\right) \equiv 0$ during the adjustment following the gate's removal) which compensates for the relaxed assumption. His general gap formula can be written as

$$
T=\frac{g^{\prime} H^{2}}{2 f}\left(1-\mathrm{e}^{v_{i w}-1}\right)^{2}
$$

where, as before, $H$ is the WBC 'potential vorticity depth' (i.e., the offshore thickness of the WBC along its eastern edge) given by Eq. (15) and $\hat{v}_{i w}$ is the initial nondimensional speed [with $\left(g^{\prime} H\right)^{1 / 2}$ ] along the wall. Namely, $v_{i w}$ is the WBC speed prior to the gate removal which is given by,

$$
v_{\mathrm{iw}}=1-\frac{1}{H}\left(-\frac{2}{g^{\prime}} \int_{0}^{L_{\mathrm{b}}} \frac{\tau}{\rho} \mathrm{d} x\right)^{1 / 2} .
$$


As before, $L_{\mathrm{b}}$ is the length of the basin; the marginal sea thickness prior to the gate's removal is taken to be zero.

Application of Eqs. (16) and (17) to our conceptual ocean is shown with the dotted line in Fig. 5. In mid latitudes the agreement with the numerical simulations is reasonable. Noting that earlier simulations of a gap on an $f$-plane (Nof, 1995) showed excellent agreement between the analytics and the numerics, we conclude that the present differences between the analytics and the numerics are primarily because of $\beta$. As already alluded to, $\beta$ breaks the front progression constraint allowing the western edge of the current to propagate further away from the gap. Consequently, more water is allowed into the marginal sea. Our numerics suggest that this $\beta$-induced increase in transport is relatively small.

As one progresses (with the marginal sea) from mid to low latitudes, the disagreement with the numerics increases dramatically. Ultimately, when the marginal sea reaches its southernmost latitude the agreement is terrible. In contrast to the $\beta$-controlled gap formula where the increase in the discrepancy (with decreasing latitude) results from friction and the breakdown of the Bernoulli, the present increase in discrepancy results from the geometry. Specifically, as we move the marginal sea southward, there is less and less room for a WBC to be established upstream of the entrance gap (BB', Fig. 2). Since such a current is an integral part of the solution leading to Eqs. (16) and (17), it is not surprising that the agreement is poor when it is not established.

\section{Minato and Kimura's barotropic transport}

Using the barotropic vorticity equation, MK derived a linear analytical solution for the exchange between an ocean and a marginal sea connected to each other via two channels. In contrast to the previous four models which allow for baroclinic effects through the $O(1)$ displacements of the interface, MK's analytical formula was derived for a strictly barotropic field with a constant depth. Furthermore, their general formulas for the transport from the ocean into a marginal sea involves frictional coefficients which, by and large, are unknown. However, in the limit of short gaps and small friction, their result reduces to the same result given by the island rule (for a barotropic flow),

$$
T=\frac{\left.g H\left(\eta_{1 \mathrm{c}}-\eta_{2 \mathrm{c}}\right)\right|_{x=0}}{f_{1}-f_{2}},
$$

where $\left.\eta_{1 \mathrm{c}}\right|_{x=0}$ and $\left.\eta_{2 \mathrm{c}}\right|_{x=0}$ are the sea-level vertical displacements along the northern and southern tips of the island (with the passages closed) and $H$ is the barotropic ocean depth.

The above formula was obtained not by integrating the equations around the island (i.e., according to Godfrey, 1989) but rather by matching a Stommel (barotropic) streamfunction solution for the ocean and a Stommel barotropic solution for the marginal sea. To see that for a barotropic fluid Eq. (18) is identical to the island rule, e.g., 


$$
\int_{\frac{B C D A}{\left(f_{1}-f_{2}\right)} \tau^{\mathrm{x}} / \rho \mathrm{d} x}
$$

we note that an integration of the linearized momentum equation

$$
-f V=-g H \frac{\partial \eta}{\partial x}+\frac{\tau^{\mathrm{x}}}{\rho}
$$

across the ocean (with the gaps closed) gives,

$$
g H \eta_{1 \mathrm{c}}-g H \eta_{2 \mathrm{c}}=\int_{B C D A} \frac{\tau^{\mathrm{x}}}{\rho} \mathrm{d} x,
$$

because

$$
\int_{A}^{B} V \mathrm{~d} x=\int_{C}^{D} V \mathrm{~d} x \equiv 0,
$$

and $U \equiv 0$ along the eastern boundary of the ocean.

Application of Eq. (18) to our conceptual ocean is shown with the dashed-doubledotted line in Fig. 5. (The application assumes that the wind stress is acting on a barotropic ocean because this is the only case to which the MK solution applies.) In equatorial and low latitudes the agreement with the numerics and the island rule is very good because the interface displacements are very small. In mid latitude, however, the agreement between Eq. (18) and the numerics is poor because the interface displacements are of $O(1)$. Like the numerical solution and the island rule, the MK solution shows a maximum transport at an intermediate latitude. However, the baroclinic effect results in the MK maximum being situated in a much higher latitude than either the island rule maximum or the numerical maximum. So much so that their solution would suggest that the transport through the Sea of Japan should be larger than the throughflow within the Caribbean [see the solid circle (corresponding to a marginal sea with a mean latitude of $22^{\circ} \mathrm{N}$, determined as the average latitude of the gaps corresponding to entering and exiting waters) and the intersection of a mean latitude line of $41^{\circ} \mathrm{N}$ with the dashed-double-dotted line]. This contradicts the four models which clearly show a much larger transport through our conceptual Caribbean (solid circle in Fig. 5) than through our conceptual Sea of Japan (dashed circle in Fig. 5). It should also be pointed out that, aside from the inadequacies involved in the applications of the MK solution to our model, there are also serious questions as to the direct application of the MK model to the ocean. This is primarily because of the relatively shallow sill in the Tsushima Strait which, in a barotropic model, would force rather large shears within the strait. 


\section{Discussion and summary}

The primary aim of our calculations was to examine the dependency of the transport entering marginal seas on the latitude of the seas. The ocean-marginal sea exchange process is viewed as being the result of opening two gates separating the two bodies of water (see gaps $\mathrm{AA}^{\prime}$ and $\mathrm{BB}^{\prime}$ in Fig. 2). Attention is focused on the final steady state resulting from the gates' removal. We used four different baroclinic models in a reduced-gravity scenario to determine the transport. [For the limitations involved in the use of a reduced gravity model (which, among other things, eliminates the possibility of having an Ekman layer right at the front) the reader is referred to Goñi, Kamholz, Garzoli and Olson (1996) and Olson (1991)].

First, we used an isopycnic model subject to an artificially magnified $\beta$ and wind stress, as well as an artificially reduced size. These modifications enabled us to conduct a relatively large number of experiments (in a reasonable amount of time) without sacrificing important details, but their downside was that the model was not eddy resolving.

Second, we used a slightly modified version of Godfrey's island rule assuming that the island $\mathrm{AB}$ (Fig. 2) is truly an island in the sense that the adjacent gaps $\mathrm{AA}^{\prime}$ and $\mathrm{BB}^{\prime}$ are as broad as the basin itself. (In our experiments, these gaps were 'only' several deformation radii broad.) The minor modification that we performed allows the upper layer to surface within the integration region.

Third, we used Nof's (1993) $\beta$-controlled formula which relates the (inviscid) transport through the entrance gap $\left(\mathrm{BB}^{\prime}\right)$ to (i) the gap distance from the WBC separation point (with the gap closed) and (ii) the upper layer thickness offshore. It is assumed in Nof's (1993) calculation that the water that leaves the basin through the entrance gap $\left(\mathrm{BB}^{\prime}\right)$ is entirely removed in the sense that it is not returned to the basin via an exit gap $\left(\mathrm{AA}^{\prime}\right)$. Furthermore, it is assumed in Nof (1993) that, since Kelvin waves require a wall on their right-hand side, no information can reach the WBC separation latitude and, therefore, it remains unaltered. Finally, it is implicitly assumed in Nof (1993) that, although the eastern boundary current initially established in the marginal sea would gradually drift westward, the drift and the current's final position have no bearing on its transport. None of these three assumptions is exactly realized in the numerical simulations. Nevertheless, they are not far from 'reality' (i.e., the numerics) and, consequently, the agreement is reasonable.

Fourth, we used Nof's (1995) general gap formula which, in contrast to Nof (1993), does not rely on the assumption that no information reaches the region northeast of the gap. Instead, it was assumed that the flow in the gap is primarily meridional and that 'a front progression condition' can be applied. It was also assumed that the transport established within a period of $O\left(f^{-1}\right)$ after the gate's removal remains the same at all subsequent times. Finally, we also plotted the transport given by MK (for short gaps and small friction) assuming that the wind stress is acting on a constant barotropic ocean instead of our 'reduced gravity' model with vertical interfacial displacements of $O(1)$. A comparison between the four different baroclinic models and the barotropic model is shown in Fig. 5. As already alluded to, we regard 
the numerical experiments as the 'truth' and examine the position of each of the other predictions relative to them.

In equatorial marginal seas (experiments 1-5, Fig. 5) there is an excellent agreement between both the island rule and the MK solution and the numerics. However, agreement is terrible between both the general gap formula and the $\beta$-controlled formula and the numerics because, in these experiments, there is no room for an upstream WBC to be established south of the gap. Such an upstream WBC is a crucial part of the solution leading to the general gap formula and, consequently, it is not surprising that the agreement is poor in its absence. The same holds for the $\beta$-controlled transport formula (i.e., it also requires an upstream WBC), except that in this case there is another assumption which is violated. This is the assumption that the Bernoulli is conserved along a streamline connecting the gap and the WBC separation. Since most of the oceanic dissipation occurs under the WBC, it is clear that this Bernoulli can only be conserved for mid-latitude gaps (i.e., gaps which are not very far from the WBC separation).

As we move with our marginal seas from low to mid latitudes (experiment 6 and on), the agreement between both the island rule and MK solution with the numerics worsens whereas the agreement between both the $\beta$-controlled gap formula and the general gap formula and the numerics improves dramatically. The difficulties with the island rule are because the zonal boundary currents are established south of the northern gap (see Figs. 3 and 4). Specifically, in these zonal boundary currents both the frictional term RU and the nonlinear terms (neglected in the derivation of the rule) are important and cannot be ignored. A similar discrepancy between the island rule and the numerics was found by Pedlosky et al. (1997). The difficulty with the MK solution is that at mid latitude the interface displacements become important. The improvement in the agreement between both the $\beta$-controlled gap formula and the general gap formula and the numerics is because a WBC is established south of the entrance gap (Fig. 4) and the proximity of the gap to the WBC separation point. Note that, despite this improvement in the agreement between the gap formulas and the numerics, and despite the worsening of the island rule prediction in high latitudes, the applications of the gap formulas to mid-latitude gaps are not really any better than the island rule is.

Before summarising our results, it is perhaps appropriate to point out again that all four baroclinic models neglect the effects of friction within the straits. This neglect is based on the idea that the straits are short compared to the length of the entire WBC, the length along which most of the oceanic dissipation occurs. To justify this neglect, it is necessary to show that the flow force across the strait $\left[\int h v^{2} \mathrm{~d} s\right]$ is much greater than the opposing frictional force associated with the bottom boundary layer. In other words, it is necessary to show that

$$
h v^{2} w \gg \frac{\tau}{\rho} w \ell
$$

where $w$ is the width of the strait, $\ell$ is the length of the strait, and $\tau / \rho=0.0016\left(u^{*}\right)^{2}$ (here $u^{*}$ is the frictional velocity). The left-hand side of Eq. (23) represents the inviscid flow force, whereas the right-hand side represent the force associated with 
the stress along the bottom. Taking $u^{*} \sim 0.1 \mathrm{~m} \mathrm{~s}^{-1}, 1 \sim O(w), w \sim 50 \mathrm{~km}, v \sim 0.7 \mathrm{~m} \mathrm{~s}^{-1}$, and $H \sim 100 \mathrm{~m}$, we find that Eq. (23) translates to $100 » 1.5$ as should be the case. This demonstrates that, as a first approximation, bottom friction can be neglected for most of the straits in question. The results of our study can be summarized as follows:

1. As the marginal sea's latitude increases from equatorial to low latitudes, the transport into the sea increases. This is because of the increased distance between the gap and the basin's southwest corner which, like all southern corners, is relatively sluggish. In other words, the increase results from an increased zonal motion immediately to the east of the southern gap and the resulting increase in the pressure difference between the gaps (which also causes an increase in the zonal pressure gradient across the marginal sea). Note that in these low latitudes, a WBC is still not established (Figs. 3 and 5) because there is a lack of room between the gap and the southern boundary.

2. As the latitude of the marginal sea increases further (i.e., from low to mid latitudes), the above tendency reverses and the transport decreases with increasing latitude. This results from the decrease in the pressure difference between the gaps and the associated decrease in the zonal pressure gradient across the marginal sea. This decrease results from (i) the establishment of a WBC south of the gap and the resulting decrease in the thickness of the upper layer along the (closed) southern gap (Figs. 4 and 5) and (ii) the fact that the pressure to which the northern gap is subjected is nearly constant because of its proximity to the WBC separation.

3. The Caribbean-Gulf of Mexico system corresponds to a low-latitude marginal sea (say, run 5 or 6 shown in Figs. 3 and 5) whereas the Sea of Japan corresponds to a mid-latitude sea (say, run 15, Figs. 4 and 5). Consequently, the transport penetrating into the conceptual Sea of Japan is about half of that penetrating into the conceptual Caribbean. (Note that although the numerics suggest a transport ratio of about one to two, the simulations were not made with the intention of duplicating in detail the actual oceans.)

4. The island rule (Godfrey, 1989) is a reasonable transport predictor for all latitudes. The primary strength of the rule is that it is very easy to use. Its primary weakness is that it does not provide any information regarding the origin of the penetrating transport; i.e., it does not tell us whether the water is coming from the north or the south. (Note that this weakness leads to a long and productive debate about the origin of the Indonesian Throughflow, e.g., Gordon, 1995; Nof 1995, 1996, 1998)

5. The general gap formula (Nof, 1995) is a reasonable predictor only for low- and mid-latitude seas; i.e., it is more limited in its application than the island rule is in the sense that it cannot be applied to very low latitudes (i.e., equatorial seas) where there is no room for the establishment of a WBC upstream. Its strength is that, in contrast to the island rule, it does tell us where the water is coming from. (This is determined from the offshore thickness in the basin and the marginal sea thickness along its eastern boundary.) Its weakness is that it is harder to use than the island rule because it involves determination of the offshore thicknesses from the wind field [compare Eqs. (16)-(18) to Eq. (13)]. 
6. The $\beta$-controlled gap formula is also reasonable but is even more limited than the general gap formula in the sense that it can be applied only to mid-latitude seas. As the general gap formula, its strength is that it tells us where the water passing through the gap originates. Its weakness is that it is more difficult to use than the island rule though it is somewhat easier to use than the general gap formula (because the initial, near-wall speed $v_{\text {iw }}$ need not be computed).

7. The agreement of both the $\beta$-controlled formula and the general gap formula with the numerics shows that the mid-latitude penetration process is inviscid in nature.

8. In equatorial and very low latitude seas, the MK barotropic solution agrees well with both the island rule and the numerics. This is because in these latitudes the vertical interface displacements are small. By contrast, in low and mid latitude the agreement between the MK solution and the numerics is poor (because of large interfacial displacements). Fig. 5 shows that the above low and mid-latitude difference between MK and the numerics is so pronounced that the MK solution is not consistent with the main conclusion of this article [stating that the transport into our conceptual Sea of Japan is significantly smaller than that into the Caribbean Sea (see solid and broken circles in Fig. 5)]. This is to say, according to MK, that the transport into the conceptual Sea of Japan needs to be greater than that into the Caribbean Sea (see the transports corresponding to the dashed-double-dotted line in Fig. 5 for seas with a mean latitude of $22^{\circ} \mathrm{N}$ and $41^{\circ} \mathrm{N}$ ).

It is hoped that this study will lead to more comprehensive global and regional numerical models which will shed more light on the processes in question.

\section{Acknowledgements}

This study was supported by the National Science Foundation under contracts OCE 9503816 and 9633655, National Aeronautics and Space Administration grants NAGW-4883, NAG5-7630 and NGT5-30164, and Office of Naval Research grant N00014-89-J-1606. Computations were executed by S. Van Gorder.

\section{References}

Arakawa, A. (1966). Computational design for long-term numerical integration of the equations of fluid motion. Two dimensional incompressible flow. Part I. Journal of Computational Physics, 1, 119-143.

Bleck, R., \& Boudra, D. (1981). Initial testing of a numerical ocean circulation model using a hybrid (quasi-isopycnic) vertical coordinate. Journal of Physical Oceanography, 11, 755-770.

Bleck, R., \& Boudra, D. (1986). Wind-driven spin-up in eddy-resolving ocean models formulated in isopycnic and isobaric coordinates. Journal of Geophysical Research, 91, 7611-7621.

Bleck, R., \& Smith, L. T. (1990). A wind-driven isopycnic coordinate model of the North and Equatorial Atlantic Ocean, 1, Model development and supporting experiments. Journal of Geophysical Research 95, 3273-3285.

Boris, J. P., \& Book, D. L. (1973). Flux-corrected transport. I. SHASTA, a fluid transport algorithm that works. Journal of Computational Physics, 11, 38-69. 
Godfrey, J. S. (1989). A Sverdrup model of the depth-integrated flow for the world ocean allowing for island circulations. Geophysical and Astrophysical Fluid Dynamics, 45, 89-112.

Godfrey, S., \& Masumoto, Y. (1999). Diagnosing the mean strength of the Indonesian Throughflow in an ocean general circulation model. Journal of Geophysical Research, 104, 7889-7895.

Goñi, G., Kamholz, S., Garzoli, S., \& Olson, D. (1996). Dynamics of the Brazil-Malvinas Confluence based on inverted echo sounders and altimetry. Journal of Geophysical Research, 101, 16273-16289.

Gordon, A. L. (1995). When is 'appearance' reality? Indonesian throughflow is primarily derived from North Pacific water masses. Journal of Physical Oceanography, 25, 1560-1567.

Hogan, P., \& Hurlburt, H. (2000). Impact of upper ocean-topographical coupling and isopycnal outcropping in Japan/East Sea models with $1 / 8^{\circ}$ to $1 / 64^{\circ}$ resolution. Journal of Physical Oceanography (in press).

Hurlburt, H. E., \& Thompson, J. D. (1980). A numerical study of Loop Current intrusions and eddy shedding. Journal of Physical Oceanography, 10, 1611-1651.

Kawabe, M. (1982a). Branching of the Tsushima Current in the Japan Sea. Part I. Data Analysis. Journal of the Oceanographic Society of Japan, 38, 95-107.

Kawabe, M. (1982b). Branching of the Tsushima Current in the Japan Sea. Part II. Numerical experiment. Journal of the Oceanographic Society of Japan, 38, 183-192.

Kim, K., \& Yoon, J. Y. (1996). Modeling of the wind-driven circulation in the Japan Sea using a reduced gravity model. Journal of the Oceanographic Society of Japan, 52, 359-373.

Minato, S., \& Kimura, R. (1980). Volume transport of the western boundary current penetrating into a marginal sea. Journal of the Oceanographic Society of Japan, 36, 185-195.

Nof, D. (1993). The penetration of Kuroshio water into the Sea of Japan. Journal of Physical Oceanography, 23, 797-807.

Nof, D. (1995). Choked flows and wind-driven interbasin exchange. Journal of Marine Research, 53, 23-48.

Nof, D. (1996). What controls the origin of the Indonesian throughflow? Journal of Geophysical Research, 101, 12301-12314.

Nof, D. (1998). The 'separation formula' and its application to the Pacific Ocean. Deep-Sea Research I, 45, 2011-2033.

Olson, D. B. (1991). Rings in the ocean. Annual Review of Earth Planetary Science, 19, $283-311$.

Pedlosky, J., Pratt, L. J., Spall, M. A., \& Helfrich, K. R. (1997). Circulation around islands and ridges. Journal of Marine Research, 55, 1199-1251.

Pratt, L. (1990). The Physical Oceanography of Sea Straits. Dordrecht (The Netherlands): Kluwer Academic.

Preller, R. H., \& Hogan, P. J. (1998). Oceanography of the Sea of Okhotsk and the Japan/East Sea. In A. R. Robinson, \& K. H. Brink, The sea (pp. 429-481). John Wiley and Sons, Inc.

Richardson, W. S., Schmitz, W. J. Jr., \& Niiler, P. P. (1969). The velocity structure of the Florida Current from the Straits of Florida to Cape Fear. Deep-Sea Research, 16, 225-231.

Schmitz, W. J. Jr. (1996). On the world ocean circulation: Volume 1: Some global features/North Atlantic circulation. Woods Hole Oceanographic Institute. Technical Report, WHOI-96-03.

Sekine, Y. (1986). Wind-driven circulation in the Japan Sea and its influence on the branching of the Tsushima Current. Progress in Oceanography, 17, 297-312.

Sekine, Y. (1988). On the seasonal variation in in- and outflow volume transport of the Japan Sea. Progress in Oceanography, 21, 269-279.

Sugimoto, T. (1990). A review of recent physical investigations on the straits around the Japanese Islands. In L. Pratt, The physical oceanography of sea straits (pp. 191-209). Kluwer Academic.

Toba, Y., Tomizawa, K., Kurasawa, Y., \& Hanawa, K. (1982). Seasonal and year-to-year variability of the Tsushima-Tsugaru Warm Current system with its possible cause. La Mer, 20, 41-51.

Wajsowicz, R. C. (1993). The circulation of the depth-integrated flow around an island with application to the Indonesian throughflow. Journal of Physical Oceanography, 23, 1470-1484.

Wajsowicz, R. C. (1994). A simple model of the Indonesian throughflow and its composition. Journal of Physical Oceanography, 23, 2683-2703.

Wajsowicz, R. C. (1996). Flow of a western boundary current through multiple straits: An electrical 
circuit analogy for the Indonesian throughflow and archipelago. Journal of Geophysical Research, $101,12295-12300$.

Yoon, J.-H. (1982a). Numerical experiment on the circulation in the Japan Sea. Part I. Formation of the East Korean Warm Current. Journal of the Oceanographic Society of Japan, 38, 43-51.

Yoon, J.-H. (1982b). Numerical experiment on the circulation in the Japan Sea. Part II. Influence of the seasonal variations of atmospheric conditions on the Tsushima Current in the Japan Sea. Journal of the Oceanographic Society of Japan, 38, 81-94.

Yoon, J.-H. (1982c). Numerical experiment on the circulation in the Japan Sea. Part III. Mechanism of the nearshore branch of the Tsushima Current. Journal of the Oceanographic Society of Japan, 38, $125-130$.

Zalesak, S. T. (1979). Fully multidimensional flux-corrected transport algorithms for fluids. Journal of Computational Physics, 31, 335-362. 